\title{
Psychosocial Determinants of Antisocial Behavior among Young Adults in Kuala Lumpur
}

\section{Mimi Fitriana a and Nur Hamizah Hj. Ramlib}

${ }^{a}$ Psychology Department, Faculty of Social Science and Liberal Art, UCSI University, Kuala

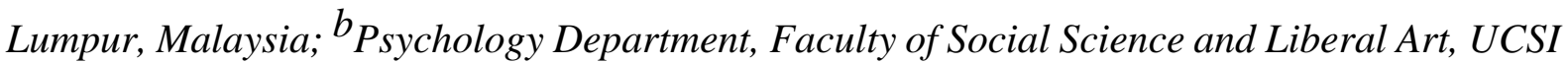
University, Kuala Lumpur, Malaysia

*Corresponding author:

Mimi Fitriana

Psychology Department

Faculty of Social Science and Liberal Art

UCSI University

Kuala Lumpur, Malaysia -56000

Email address: mimifitrianam@ucsiuniversity.edu.my 


\title{
Psychosocial Determinants of Antisocial Behavior among Young Adults in Kuala Lumpur
}

\begin{abstract}
The current research focuses on the antisocial behaviors which lead to juvenile delinquency. The current study looks at young adults in Kuala Lumpur and their perceptions of the typical characteristics of conduct disorders, substance abuse, and thought and attention problems commonly found among juvenile delinquents. This qualitative research study is aimed at investigating the psychosocial determinants of antisocial behaviors. After a purposive sampling procedure, a semi-structured interview was conducted with two young adult females. In-depth analysis was used to explore their narratives of lived experience. Themes were generated and analyzed through the procedure of thematic analysis. The findings revealed that major psychosocial factors that lead to young people's antisocial behaviors include 1) aggressive behavior, 2) poor psychosocial growth, 3) societal negligence, 4) grandiose thinking, 5) dysfunctional thoughts, 6) compromised intrafamilial relationships, 7) encouragement of violence by peers, and 8) repression of individuals' inner emotional expressions antisocial. Implications of the current research are discussed in terms of producing early identification of antisocial behaviors for the purpose of developing appropriate interventions.
\end{abstract}

Keywords: antisocial behavior, psychosocial determinants, adolescent behavior, intrafamilial relationships

\section{Introduction}

From its earliest days, society has been concerned with managing misbehavior in children and young adults (Chingtham, 2015). When young adults engage in antisocial behavior they act in ways that are meant to cause annoyance, damage, or harm to others. Such behavior often leads to juvenile delinquency as well. Juvenile delinquency is a complex social problem that significantly affects all members and processes of society (Jones, 2014). The various factors leading to delinquency, have similar underlying themes. These include reference to physical and mental factors, home conditions, school and neighborhood environments, and occupational situation (Arseneault, et al., as cited in Howell, 2015).

In a study by Chingtham (2015), the causes of juvenile delinquency were investigated in 120 juvenile delinquents in Manipur, India. The study revealed that social norms and values played a major role in contributing to the development of juvenile delinquency. The moral confusion, increase in substance abuse, decay in values, and changes in norms prevalent in modern society have negatively affected the youth and have contributed to their delinquency. Family factors contributing to the development of juvenile delinquency include coming from a broken family, living in poverty and scarcity, disabled parents, absence of ethical norms, good values and proper discipline, antisocial behavior. Meanwhile, the personal factor focuses on their wellbeing and genetic hereditary factors only. Poor mental health will also lead to the development of juvenile delinquency. Additionally, if the parents are involved in criminality, there is a greater chance that their children will be involved too. Psychological factors such as mental disorders, 
imbalance personality, emotional conflicts, intolerance for ambiguity, instability and such other negative factors contribute significantly to the development of delinquent behavior. Finally, academic factors such as the atmosphere of the school, both inside and outside the classroom, numerous cases of dropouts, disinterestedness in academic activities, peer groups, parental neglect and rejection and teachers indifferences toward their students turn them to be deviant and delinquents.

Conduct disorder is the most common mental health problem seen in juvenile delinquents. Unfortunately, it is often misunderstood in the context of the juvenile justice system (Frick, 2015). Numerous studies conducted to find out how conduct disorder affects behavior have pointed out that certain types of mental disorders are common among adolescent and young adult offenders. Some of the symptoms of these disorders appear to increase their risk of engaging in aggressive behaviors as well (Teplin, Abram, McClelland, Dulcan \& Mericle, 2002; Wasserman, Mcreynolds, Lucas, Fisher \& Santos, 2002; Atkins et al., as cited in Underwood \& Washington, 2016).

Stoddard-dare, Mallett and Boitel (2011) studied a sample of 341 juveniles randomly drawn from a U.S. juvenile detention facility to investigate which specific mental health disorders predicted detention for committing a crime. There was a significant relationship found between mental health difficulties such as conduct disorder, ADHD, and bipolar disorder in adolescents and the crimes that they commit. This study highlighted that adolescents with bipolar disorder are more likely than their peers to commit crime. The researchers found that adolescents with bipolar disorder were unable to handle these symptoms and instead they acted out because of the challenging "high highs and low lows" (Stoddard-dare, Mallett \& Boitel, 2011).

In their study of 51 adolescents with delinquent behavior, Jurma, Tocea, Iancu, Ciocani and Nache (2014) investigated the presence of psychiatric symptoms in adolescents with delinquent behavior in Tiomiosara, Western Romania. The study revealed a strong correlation between psychiatric symptoms and delinquent behavior. This study is supported by the research of Mbelwa (2017) on 108 juvenile delinquents. This mixed method study was carried out to investigate the presence of mental health disorders and associated factors. These juvenile delinquents revealed that they were facing problems with substance abuse, depression, brief psychotic reactions, sexual activities, and ADHD. Based on this study, other factors contributing to the commission of offenses were difficulties in their psychosocial environment such as parental death and upbringing by a single parent. In some cases, there were major problems with parental care, which resulted in these youth becoming street children, who committed offenses because they needed to survive day-to-day.

Muscari and Ngo (2017) found that children with ADHD paired with conduct disorder or oppositional defiant disorder were more likely to commit delinquent acts than those with ADHD alone. However, ADHD on its own, especially when accompanied by parental criminality and sibling delinquency, can lead to negative behaviors also. Children with untreated ADHD tend to engage in behaviors that disrupt their family life, schooling, and peer relationships. Comorbidities such as substance abuse and conduct disorder are common in ADHD, and these 
make it more likely that ADHD may contribute to criminal behaviors. Dan et al., (2015) studied 42 children diagnosed with conduct disorder in Romania to evaluate the level of emotional and behavioral problems in adolescents diagnosed with conduct disorder. The study revealed that the most common comorbidity in conduct disorder is ADHD, followed by major depression, substance abuse, and anxiety disorders.

Regarding a Malaysian perspective, only a few studies related to the topic juvenile delinquency and conduct disorder were found. Aida et al., (2014) conducted a study on 105 detainees to determine the presence of psychiatric disorder and their association with personal characteristics of juvenile detainees. Conduct disorder was the most common disorder found. A significant correlation was also found between substance abuse and conduct disorder. Badyai, Khairudin, Ismail, and Sulaiman (2016) studied 404 juvenile delinquents examining their symptoms of problem behaviors. Thought problems and attentional problems were found to be common in these juvenile delinquents. This indicated that these young offenders might have issues with their decision-making and problem-solving abilities, causing them to engage in antisocial behavior.

Conduct disorders are the most common mental health disorders of childhood and adolescence and the most common reasons for referral to child and adolescent mental health services in Western countries, and, thus, most studies related to conduct disorder and juvenile delinquency focus on the Western countries, but not on Asia, especially Malaysia. Mental health issues are still highly trivialized and highly stigmatized in Asian cultures (Ling, 2017). In Asian cultures, mental illness is often associated with weakness, guilt, and shame, often resulting in the dismissal of any issues related to mental illness in every facet of Asian lives. This study seeks to break this silence and to bring about in-depth understanding of antisocial behavior and mental health among young adults in Kuala Lumpur and the rest of Asia.

\section{Methodology}

The research design adopted for this study is a qualitative research narrative. This approach weaves together a sequence of events told from the viewpoint of two individuals to form a cohesive story (Sauro, 2015). The participants were contacted through a purposive sampling method, whereby they admitted to having a history of behavioral problems. This research called for young adults in Malaysia aged 18-25 years old who had a record of behaving antisocially. The data were collected through interviews using 19 open-ended, semi-structured questions developed based on the literature.

Due to the fact that the participants in this study were from a risk-sensitive population, the sample was identified to be prone to act out (Nugent, 2013) aggressive behavior if they are being triggered by an externalizing factor. Prior to conducting the study, the researcher had to make the participants understand that they had all the right to withdraw at any time when they felt uncomfortable in the process of doing this research and that their identity would be kept confidential. The review board of the psychology department of UCSI University approved the study protocol to ensure adherence to ethical principles. 
The interview data was then analyzed with using narrative analysis through the development of open coding, axial coding, and thematic analysis based on the transcribed interview.

\section{Results}

There are about eleven themes generated through coding for (1) perception of antisocial behavior by the antisocial behaving individuals, (2) perception of the occurrence of conduct disorder toward antisocial behavior by the antisocial behaving individual, (3) perception of the antisocial behaving individual on the attribution of the occurrence of antisocial behavior (4) perception of the antisocial behaving individual on the influence of the occurrence of antisocial behavior. This study revealed that the major factors that lead them to behave antisocially were aggressive behavior, poor psychosocial growth, societal ignorance grandiose thinking, dysfunctional thoughts, lack of interfamilial relation, peer violence encouragement and individual violent expression.

\section{Aggressive Behavior}

Both respondents reported that they intentionally showed aggressive behavior because of their own inner frustration. They reported harming other people and damaging public facilities. When it comes to hurting other people, the respondents reported that they usually do it because they have got nothing else to do and that once they get bored with one victim, they will find another person to hurt. It goes in a cycle. One of the respondents said that this behavior is the result of childhood experiences and the problems within the environment where she grew up.

\section{Thinking Only of Themselves}

According to the respondents, hurting other people or damaging public facilities was often done as a form of self-expression and to relieve their inner frustration. According to the respondents, they barely think about the people they are harming when they act out behaviors to relieve their inner frustration. They only think about themselves. They do not think about other people's feelings or how much distress they are causing for their victims. They just want to fulfill their desire to act out.

\section{Poor Psychosocial Growth}

The respondents agreed that their bad behavior usually came from the pressures and problems of their surroundings. For instance, since the area where they have grown up is a well-known crime area, there was a greater tendency for them to behave as criminals. Another problem with their environment is no one ever told them that their behavior was morally unacceptable, and since everyone else was acting similarly, they perceived what they were doing as socially acceptable.

\section{Lack of intrafamilial Relation}

A lack of familial relationships is closely associated with poor family guidance, lack of communication, and decreased familial attachment. When the youth is lacking all these, it leads them to purposely act out. Since these young adults have less attachment, they never think of the impact of their actions on their family. Lack of familial communication also leads these 
young people to act out. Since the families barely talk and do not even make simple conversation such as asking, "Hey, how was your day?," how can the families guide and support these young adults when they barely even talk to each other?

Another thing about this group of people is that, they usually are punished for behaving the way they are. Instead, of understanding why, the punishers ignored them further, which then creates intense sense of isolation for these individuals. This will further encourage the antisocial behaving individuals to act out even more, which worst comes to worst, them to be a juvenile delinquent.

\section{Inner Emotional Expression}

Because they are struggling inside emotionally, most young adults do not understand their emotional upheavals and they are afraid to confide in friends, family members, or anyone else. Due to their fears, they instead choose to act out in the hope that people around them will notice that there is a problem and will try to do something about it.

\section{Societal Ignorance}

When one grows up in a secluded environment, one tends to get subsumed within the majority's mindset and to behave as they behave. According to Kelman (1958), when an individual accepts influence because the content of the induced behavior is consistent with the individual's value system, it is called internalization. In this situation, it becomes a firm part of the individual's behavioral repertoire because, again, no one has told them what they are doing is unacceptable socially and morally.

\section{Dysfunctional Thoughts}

Because antisocial individuals often have actually gone through a difficult period in their past and have never got the chance to have closure, they might be suffering from trauma with their inability to cope with that part of their past, and they chose to pass on the pain to someone else. This is a sign of dysfunctional thoughts.

\section{Grandiose Thinking}

Grandiose thinking involves having an unrealistic sense of being special and better than other people. This grandiosity is common in people with antisocial behavior. According to one of the respondents, antisocial individuals might feel worthless, and, therefore, they want to feel superior. He went on to say that to divert the feeling of worthlessness, they could pick on someone so that that person is affected by the antisocial individual's actions. Thus, if a victim is affected by their actions and becomes afraid of them, the perpetrator will be enabled to develop a feeling of superiority. According to one respondent, a reason why she continued acting out is because it gave her a sense of satisfaction and a feeling of superiority. To her, what she was doing was out of the ordinary, and it made her different, making her stand out from the crowd. 


\section{Poor Psychosocial Growth}

According to one of the respondents, the reason she kept doing socially unacceptable actions is although the people around her notice when she was acting out, they do not do anything to stop her. This has led her to thinking that the people around her did not care about her and what she does; therefore, she felt it was okay to continue her antisocial functioning.

\section{Dysfunctional thoughts}

The main thing that these two respondents have in common is they did not think much when they acted out; they just went ahead with it.

\section{Self-Demand Character}

When an individual has no positive self-regard, no self-regulation, and no self-efficacy, they experience a lack of self-worth, and they often are not aware of what they are doing. Often, this kind of thing would eventually lead to the development of bipolar disorder, where they will have grandiose thinking: feeling of superiority.

\section{Violence Toward Self}

Lack of emotional support from their loved ones was also seen as a factor that contributed to the participants' antisocial behavior. One respondent had shown suicidal tendencies because she could not get anyone to comfort her. It was her way of expressing her frustrations and at the same time "teaching" the people around her what would it be like to lose her.

\section{Peer Encouragement of Violence}

One respondent revealed that she was influenced by challenges from her friends to do violent things.

\section{Purposeful Violence Exposure}

Another thing that these two respondents have in common is, their actions are intended to attract attention. As one respondent revealed, if she is in a large group, it is likely she will do destructive things to impress people and to hype up her feelings of superiority. The other respondent also acted out to attract attention; however, she sought it from her loved ones rather than from her peers. It is as a result of her internal frustration.

\section{Lack of Intrafamilial Interaction}

In the ever-changing society, most parents these days are both working parents, compared to yesteryears. To have the life that they have dreamed of giving their children, they have sacrificed everything, including their interaction time with their children. However, this can have a negative impact on their children's mental and emotional development. When parents are swamped with work, they miss out on the things going on in their children's lives such as their academic achievements, their social interactions, and their favorite activities. Less interaction time is equivalent to less attention given to the children, and when children are not nurtured properly, they are more likely to become antisocial. 


\section{Parental Restriction}

Often, negative parenting style such as authoritarian parenting and neglectful parenting have a problematic effect on children. According to Lokoyi (2015), authoritarian parents are not only demanding, but they are also unresponsive. Moreover, being too strict with young children may result in behavior problems in adolescence. These shared parenting styles may not support children and their emotional needs. The parents may be seen as just caring about themselves and their own demands. Children who have this kind of parenting often have difficulties in expressing themselves, and they struggle with their emotions. This can lead to numerous problems, including mental illness.

One of the outcomes of having no one around to tell them that what they are doing is wrong, is the behavior becomes a habit. A habit that they cannot stop because it does not feel right if they do not do it. One of the respondents mentioned that she broke the rules or acted out antisocially because the thoughts of not doing it, will bother her. To her, to shut her thoughts up is by doing exactly what the thoughts said or else it will disrupt her daily functioning and bother her till she has really done it.

\section{Peer Violence Encouragement}

One of the respondents revealed that her friends are also involved in rule-breaking behavior. From here, it can be seen that peer pressure has played a role in the respondents' antisocial functions.

\section{Discussion and Conclusion}

Psychosocial factors have contributed to the occurrence of antisocial behavior in young people. To understand the outbreak of antisocial behavior in young adults further, it is recommended that future longitudinal studies are done on how psychosocial growth affects long-term behavior. Examining the effects of cultural variations (from collective-oriented to individualistic-oriented) on young adults' delinquent and antisocial behavior is also recommended to analyze cultural variations.

Based on the thematic analysis, both respondents showed symptoms of bipolar disorder, with dysfunctional thoughts and grandiose thinking. This finding is consistent with previous studies where individuals with a mental illness such as bipolar disorder are unable to handle their symptoms and they act out (Stoddard-dare, Mallett \& Boitel, 2011).

The findings of the current study further suggest thoughts pattern which are consistent with Badyai, Khairudin, Ismail, and Wan Sulaiman's (2016) finding that thought and attention problems are common in juvenile delinquents. This indicates that these young offenders might have issues with their decision-making and problem-solving abilities which lead them into antisocial behavior.

It has been identified that poor psychosocial growth, dysfunctional thoughts, self-demand character, purposeful exposure to violence, grandiose thinking, individual expressions of 
violence, and peer encouragement of violence contribute to the occurrence of antisocial behavior the result of this study leans heavily toward social factors as causative agents. Chingtham (2015) also showed that social factors play a major role in contributing to the development of juvenile delinquency. Additionally, based on the interviews with the study participants, the findings suggest that, overly restrictive and overworked, under-involved parenting affect children's wellbeing in ways that lead them to behave antisocially out of frustration.

\section{Research Implication and Recommendation}

The current research makes a significant contribution to the occurrence of antisocial behaviors among youth. Findings of the research indicated the development of further analysis of youth's behavior which involved aggressiveness and other types of antisocial behavior. It might assist researchers, therapists, parents, or peers who are dealing with individuals with antisocial behavior to come up with a strategic plan to help them in coping with their inner struggles.

To understand the outbreak of antisocial behavior in young adults further, it is recommended to do longitudinal studies on how psychosocial growth affects behavior over time. Examining the effects of cultural variations (from collective-oriented to individualistic-oriented) on young adults' antisocial behavior is also highly recommended.

\section{References}

Badyai, A. R. A., Khairudin, R., Ismail, K. H., \& Sulaiman, W. S. W. (2016). An Exploratory Study on Symptoms of Problem Behaviors Among Juvenile Offenders (1st ed.). Jurnal Psikologi Malaysia, 30, 69-79.

Aida, S., Aili, H., Manveen, K., Salwina, W., Subash, K., Ng, C., \& Muhsin, A. (2014). Prevalence of psychiatric disorders among juvenile offenders in Malaysian prisons and association with socio-demographic and personal factors. International Journal of Prisoner Health, 10, 132-143.

Atkins, D., Pumariega, A., Rogers, K., Montgomery, L., Nybro, C., Jeffers, G., \& Sease, F. (1999). Mental health and incarcerated youth. I: Prevalence and nature of psychopathology. Journal of Child and Family Studies, 8, 193-204.

Chingtham, T. (2015). Causes of Juvenile Delinquency in the Higher Secondary School Students. IOSR Journal of Research \& Method in Education, 5, 20-24.

Clay, C. (2017). Types of Juvenile Delinquency Crimes. Soapboxie. Retrieved from https://soapboxie.com/government/Juvenile-Delinquency-Crimes.

Connor D. (2002). Aggression and Antisocial Behavior in Children and Adolescents. New York, NY, USA: Guilford Press.

Department of Statistics Malaysia. (2017). Dosm.gov.my. Retrieved from https://www.dosm.gov.my/v1/index.php?r=column/ctheme\&menu_id=U3VPMldoYUxzVzFaYmNkWX ZteGduZz09\&bul_id=NVYwaEtwM21MempVbWpBZFpxOFZHZz09.

Frick, P. (2015). Conduct Disorder: Recent Research and Implications for Serving Children and Adolescents in The Juvenile Justice System. New Orleans: National Courts and Science Institute, Inc. Retrieved from https://sites01.lsu.edu/faculty/pfricklab/wp-content/uploads/sites/100/2015/11/SCR-Frick-ConductDisorder-in-JJS.pdf.

Howell, L. (2015). Causes of Juvenile Delinquency. A Literature Review, Munich, GRIN Verlag. Retrieved from http://www.grin.com/en/e-book/310357/causes-of-juvenile-delinquency-a-literature-review.

Jones, D. (2014). Trends in Juvenile Delinquency (Master's thesis). Northern Michigan University, USA. 
Jurma, A., Tocea, C., Iancu, O., Ciocani, M., \& Enache, A. (2014). Psychopathological symptoms in adolescents with delinquent behavior. Romanian Journal of Legal Medicine, 22, 193-198.

Lim, B. (2017). Mental Health Council sounds alarm bell on M'sia's psychiatrist shortage. NST Online. Retrieved from https://www.nst.com.my/news/2017/01/206598/mental-health-council-sounds-alarm-bell-msiaspsychiatrist-shortage.

Ling, C. (2017). Societal Views on Mental Health in Asia Need to Change. The Huffington Post. Retrieved from https://www.huffingtonpost.com/christina-ling/societal-views-on-mental-_b_9488896.html.

Lokoyi, O. L. O. (2015). Parenting styles as correlates of aggressive behaviour among in-school adolescent with mild intellectual disability. Psychology and Behavioral Sciences, 4, 94-100.

Mbelwa, J. S. (2017). Mental disorders and associated factors among adolescents in juvenile detention, Dar es Salaam, Tanzania. Open Journal of Nursing, 07, 993-1020.

Muscari, M., \& Ngo, H. (2017). Attention deficit hyperactivity disorder (ADHD). The Encyclopedia of Juvenile Delinquency and Justice, 1-5.

Novaco R. (1994). Anger as a Risk Factor for Violence among the Mentally Disordered. In J. Monahan, H. Steadman (Ed.), Violence and Mental Disorder: Development in Risk Assessment (pp. 21-59). Chicago, IL, USA: University of Chicago Press.

Nugent, P. (2013). What is risk sensitivity? Definition of risk sensitivity (Psychology Dictionary). Psychology Dictionary. Retrieved from https://psychologydictionary.org/risk-sensitivity/.

Nugent, P. (2013). What is psychosocial factors? Definition of psychosocial factors (Psychology Dictionary). Psychology Dictionary. Retrieved from https://psychologydictionary.org/psychosocialfactors/.

Roberts, C. (2017). Juvenile delinquency: Cause and effect. Teachersinstitute.yale.edu. Retrieved from http://teachersinstitute.yale.edu/curriculum/units/2000/2/00.02.05.x.html.

Sauro, J. (2015). MeasuringU: 5 Types of Qualitative Methods. Measuringu.com. Retrieved from https://measuringu.com/qual-methods/.

Schlenker, M. (2017). SOCHUM II: Juvenile Delinquency Around the World. Shorthand. Retrieved from https://social.shorthand.com/ymuntaiwan/3yJT67aWhT/sochum-ii-juvenile-delinquency-around-theworld.

Shek, D., \& Lin, L. (2016). Delinquent behavior in high school students in Hong Kong: Sociodemographic, personal, and family determinants. Journal of Pediatric and Adolescent Gynecology, 29, S61-S71.

Stoddard-Dare, P., Mallett, C., \& Boitel, C. (2011). Association between mental health disorders and juveniles' detention for a personal crime. Child and Adolescent Mental Health, 16, 208-213.

Stephens, C., \& Breheny, M. (2012). Narrative analysis in psychological research: An integrated approach to interpreting stories. Qualitative Research in Psychology, 10, 14-27.

Tamura, M., Moriguchi, Y., Higuchi, S., Hida, A., Enomoto, M., Umezawa, J., \& Mishima, K. (2012). Neural network development in late adolescents during observation of risk-taking action. PLoS ONE, 7, e39527.

Teplin, L., Abram, K., McClelland, G., Dulcan, M., \& Mericle, A. (2002). Psychiatric disorders in youth in juvenile detention. Archives of General Psychiatry, 59, 1133.

The American Academy of Child and Adolescent Psychiatry. (2013). Conduct disorder. Retrieved from http://www.aacap.org/AACAP/Families_and_Youth/Facts_for_Families/FFF-Guide/Conduct-Disorder033.aspx

Underwood, L., \& Washington, A. (2016). Mental illness and juvenile offenders. International Journal of Environmental Research and Public Health, 13, 228.

Wasserman, G., Mcreynolds, L., Lucas, C., Fisher, P., \& Santos, L. (2002). The voice DISC-IV with incarcerated male youths: Prevalence of disorder. Journal of The American Academy of Child \& Adolescent Psychiatry, 41, 314-321. 